\title{
Characterization of Quasi-Concave Functions and Its Optimality Conditions In $\square^{N}$
}

\author{
Efor, T. E (Mrs) and U.A Osisiogu \\ Department of Industrial Mathematics and Applied Statistics Ebonyi state University, Abakaliki.
}

\begin{abstract}
Let $T$ be open, nonempty subset of $\square^{n}$ and $f: T \rightarrow \square$ be a function on $T$. In general if $f$ is concave, every local maximum of $f$ is also a global maximum and first order conditions are sufficient to identify global of concave or convex optimization problems and they are so strict and quite restrictive as an assumption.

In this paper we present characterizations of optimization problems under a weakening of the condition of concave functions, called "quasi-concave" functions. Some of the characterizations of these functions as a generalization of concave functions and proves are discussed in terms of its contour sets (level sets), derivatives and extreme properties of the functions on line segment. It also discuses its optimality conditions
\end{abstract}

Key words: Concave functions, quasi-concave functions, Semi-strict, Upper and lower contour.

\section{Introduction}

Concave and convex functions have been studied by some authors like Finetti (1949), Mangasarian, Jensen, Mordecai Avriel-Diewert-Schaible-Zang, Sundaram and so many others. Due to the beautiful works done by Fenchel, Moreau, Rockafellar in 1960s and 1970s, Concave analysis became one of most developed branches of mathematics. It has a wide range of applications in optimization, operations research, economics etc. However several practical problems involve functions which are not exactly convex, but share certain properties of concave functions. These functions are generalization of concave functions and are called quasiconcave functions. These functions fail to exhibit many of the sharp properties that distinguish concave and convex functions, such as; a quasi- concave or convex function may, for instance, be discontinuous on the interior of its domain. A local maximum of a quasi-concave function need not also be a global maximum of the function. More significantly, first order conditions are not in general sufficient to identify global of quasiconcave or convex optimization problems.

However, quasi- concave and quasi convex functions posses enough structure that is important for optimization theory, for instance, it turns out that the Kuhn-Tucker first order condition are almost sufficient to identify optima of inequality constrained optimization problems under quasi-concavity restrictions, more precisely, the first order conditions are sufficient provided the optimum occurs at a point where an additional regularity condition is also met.

\subsection{Concave, Quasi-concave and Pseudo-concave}

A function $f: T \subset \square^{n} \rightarrow \square$ is concave on $T$ if subgraph of $f$ at $\alpha \in \square$

Sub. $f=\{(x, \alpha) \in T \times \square: f(x) \geq \alpha\}$

is a convex set.

A function $f$ is said to be convex on $T$ if epigraph of $f$ at $\alpha \in \square$

$$
\text { epif }=\{(x, \alpha) \in T \times \square: f(x) \leq \alpha\}
$$

is a convex set

A function $f: T \rightarrow \square \subset \square^{n}$ is said to be concave if

$f(\lambda x+(1-\lambda) y) \geq \lambda f(x)+(1-\lambda) f(y) \forall x, y \in T, \lambda \in(0,1)$

If the inequality in (1.3) is strict, and, $x \neq y$, then the function is called a strict concave function.

1.3 Characterization of Quasi-concave in terms of Contour (Level) sets.

Here, we characterize quasi-concave using their contour sets: 
Let $f: T \subset \square^{n} \rightarrow \square$, where $T$ is convex set, $f$ is said to be quasi-concave on $T$ if the upper contour set of $f$ at $\alpha \in \square$

$\bigcup_{f}(\alpha)=\{x \in T: f(x) \geq \alpha\}$

is a convex set, similarly, $f$ is said to be quasi-convex on $T$ if the lower-contour set of $f$ denoted by $L_{f}(\alpha)$ for each $\alpha$

$$
L_{f}(\alpha)=\{x \in T: f(x) \leq \alpha\} \text {. }
$$

is a convex set.

Theorem 1.1 Let $f: T \subset \square^{n} \rightarrow \square, f$ is said to be quasi- concave if and only if for all $x, y \in K$ and $\lambda \in(0,1)$

$$
f(\lambda x+(1-\lambda) y) \geq \min \{f(x), f(y)\}
$$

It is quasi-convex if and only if for all $x, y \in K \quad \lambda \in(0,1)$

$$
f(\lambda x+(1-\lambda)) y \leq \max \{f(x), f(y)\}
$$

We proof first part and second part can easily be obtained by recognizing that $f$ is quasi- convex if and only if $-f$ is quasi- concave.

\section{Proof}

First, suppose that $f$ is quasi- concave. Then $U_{f}(\alpha)$ is a convex for each $\alpha \in \square$. Let $x \in T, y \in T$ and $\lambda \in(0,1)$. Assume, without loss of generality, that, $f(x) \geq f(y)$, letting $f(y)=\alpha$, we have $x \in U_{f}(\alpha), y \in U_{f}(\alpha)$. By convexity of $U_{f}(\alpha)$, we have $\lambda x+(1-\lambda) y \in U_{f}(\alpha)$

$\Rightarrow f(\lambda x+(1-\lambda)) y \geq f(y) \geq \alpha=f(y)=\min \{f(x), f(y)\}$.

Conversely, suppose $U_{f}(\alpha)$ is convex. Let $\alpha \in \square$, if $U_{f}(\alpha)$ is empty or contains only one point, it is clear that it is convex, but suppose it contains at least two points $x$ and $y$, then $f(x) \geq \alpha$ and $f(y) \geq \alpha$, so $\min \{f(x), f(y)\} \geq \alpha$

$$
f(\lambda x+(1-\lambda) y) \geq \min \{f(x), f(y)\},
$$

by hypothesis, and so $\lambda x+(1-\lambda) y \in U_{f}(\alpha)$. Hence $U_{f}(\alpha)$ is a convex set and $f$ is quasi concave.

Quasi-concave function is a generalization of concave functions since we can show that the set of all quasi-concave functions contains the set of all concave functions.

Theorem 1.2: Let $f: T \subset \square^{n} \rightarrow \square$. If $f$ is concave on $T$ it is also quasi concave on $T$. If $f$ is convex on $T$, it is also quasi-convex on $T$.

We prove the first part of the theorem and the second part will be proved analogously.

\section{Proof:}

Suppose $f$ is concave, then, for all $x, y \in T$ and $\lambda \in(0,1)$ we have.

$$
\begin{aligned}
f(\lambda x+(1-\lambda) y) & \geq \lambda f(x)+(1-\lambda) f(y) \\
\geq & \lambda \min \{f(x), f(y)\}+(1-\lambda) \min \{f(x), f(y)\} \\
= & \min \{f(x), f(y)\}
\end{aligned}
$$

Suppose $f$ is concave, then for all $x, y \in T$ and $\lambda \in(0,1)$

We have $f(\lambda x+(1-\lambda) y) \geq \lambda f(x)+(1-\lambda) f(y)$ 


$$
\begin{aligned}
& \geq \lambda \min \{f(x), f(y)\}+(1-\lambda) \min \{f(x), f(y)\} \\
& =\min \{f(x), f(y)\} .
\end{aligned}
$$

The example below shows that the converse of this result is not true.

\section{Example 1.1}

Let $f: \square \rightarrow \square$ be any non-decreasing function on $\square$. Then $f$ is quasi-concave and quasi-convex on $\square$. It is always possible to choose a non-decreasing function that is neither concave nor convex on $\square$ (take for instance $f(x)=x^{3} \forall x \in \square$ ). This shows that not every quasi-concave function is concave and not every quasi-convex function is convex.

Theorem 1.3: If $f: T \rightarrow \square$ is quasi-concave on $T$ and $\varphi: \square \rightarrow \square$ is monotone convex non-decreasing function, then the composition $\varphi \circ f$ is a quasi concave function from $T$ to $\square$. In particular any monotone transform of a concave function results in a quasi-concave function.

\section{Proof}

Pick any $x$ and $y \in K, \lambda \in(0,1)$ we show that

$$
\varphi(f([\lambda x+(1-\lambda) y])) \geq \min \{\varphi(f(x)), \varphi(f(y))\}
$$

Since $f$ is quasi-concave by hypothesis, we have

$$
f([\lambda x+(1-\lambda) y]) \geq \min \{f(x), f(y)\}
$$

Since $\varphi$ is non-decreasing, it implies that

$$
\varphi(f([\lambda x+(1-\lambda) y])) \geq \varphi(\min \{(f(x)),(f(y))\})=\min \{\varphi(f(x)), \varphi(f(y))\}
$$

\subsection{Implications of a quasi-concavity}

Although the upper contour(level) sets of both concave and quasi-concave functions are convex, quasiconcave functions can considerably differ from concave functions, for instance, quasi-concave functions can be discontinuous in the interior of their domain, not every local maximum is a global maximum, local maxima that are not global cannot be strict maxima. First order conditions are not sufficient to identify even local optima under quasi-concavity.

The example below explain these implications

Example 1.2 Let $f: \square \rightarrow \square$ be defined by

$$
f(x)=\left\{\begin{array}{cc}
x^{3} & x \in[0,1] \\
1 & x \in(1,2] \\
x^{3} & x>2
\end{array}\right.
$$

This function is a non-decreasing function, it is both quasi-concave and quasi convex on $\square$.Clearly, $f$ has a discontinuity at $x=2, f$ is constant on the open interval $(1,2)$, so every point in this interval is a local maximum of $f$ as well as local minimum of $f$. However, no point in $(0,1)$ is either a global maximum or a global minimum. Lastly $f^{\prime}(0)=0$ and 0 is neither a local maximum nor a local minimum.

More over, distinction between convexity and quasi-convexity arises from the fact that a strictly convex function cannot be even weakly concave and a strictly concave function cannot be even weakly convex. However, strictly quasi concave function may as well be strictly quasi convex.

Example 1.3: If $f$ is strictly increasing function on $\square$. That is if $x>y$ implies $f(x)>f(y)$ then $f$ is both strictly quasi-concave and strictly quasi-convex on $\square$.

Theorem 1.4 Let $f$ be a quasi-concave function defined on the convex set $T \subset \square^{n}$. 
If $x^{*} \subset T$, is a strict local maximum of $f$. Then $x^{*}$ is also a strict global maximum of $f$ on $T$. The set of points at which $f$ attains its global maximum over $T$ is a convex set.

Now, we characterize quasi-concave functions in terms of its derivatives.

\subsection{First Derivative Characterization of Quasi-concave functions.[6]}

Theorem 1.5: Let $f: T \rightarrow \square$ be a differentiable function on $T$, where $T \subset \square^{n}$ is convex and open. Then $f$ is quasi- concave on $T$ if and only if $\forall x \in T, y \in T, f(x) \geq f(y) \Rightarrow \nabla f(y)(x-y)^{T} \geq 0$

$(1.12)$

Proof:

Suppose first that $f$ is quasi-concave on $T$, then

$f(x) \geq f(y) \Rightarrow f(\lambda x+(1-\lambda) y) \geq f(y)$ for

$\lambda \in(0,1)$, since $f$ is quasi-concave, we have

$f(y+\lambda(x-y))=f((1-\lambda) y+\lambda x) \geq \min \{f(x), f(y)\}=f(x)$

Therefore, for all $\lambda \in(0,1)$

$$
\frac{f(y+\lambda(x-y))-f(y)}{\lambda} \geq 0
$$

As $\lambda \rightarrow 0^{+}$, that is, taking the limit as $\lambda$ approaches through positive numbers, we obtain $\nabla f(x)(x-y)^{T} \geq 0$, proving one part of the result.

\section{Conversely}

Assume that for all $x, y \in T$ such that $f(y) \leq f(x)$, we have

$\nabla f(x)(y-x) \geq 0$. Pick any $x, y \in T$, and suppose without loss of generality that $f(x)=\min \{f(x), f(y)\}$. We show that for any $\lambda \in(0,1)$, we also have $f[(1-\lambda) x+\lambda y] \geq \min \{f(x), f(y)\}$,

establishing the quasi- concavity of $f$.

\subsection{Second derivative characterization of quasi concave functions}

We give a second - derivative test for quasi concave functions. Let a twice continuous differentiable, $C^{2}$ function $f$ defined on some open domain $T \subset \square^{n}$ be given, and let $x \in T$, let $A_{t}(x) t=1, \ldots, n$, be the $(t+1) \times(t+1)$ matrix defined by

$$
A_{t}(x)=\left[\begin{array}{ccc}
0 & \frac{\partial f(x)}{\partial x_{1}} \cdots & \frac{\partial f(x)}{\partial x_{t}} \\
\frac{\partial f(x)}{\partial x_{1}} & \frac{\partial^{2} f(x)}{\partial x_{1}^{2}} \cdots & \frac{\partial^{2} f(x)}{\partial x_{1} \partial x_{t}} \\
\vdots & \vdots & \vdots \\
\frac{\partial f(x)}{\partial x_{t}} & \frac{\partial^{2} f(x)}{\partial x_{t} \partial x_{1}} \cdots & \frac{\partial^{2} f(x)}{\partial x_{t}^{2}}
\end{array}\right]
$$

Theorem 1.6: Let $f: T \rightarrow \square$ be a $C^{2}$ function, where $T \subset \square^{n}$ is open and convex. Then:

(1) If $f$ is quasi- concave on $T$, we have If $(-1)^{t}\left|A_{t}(x)\right| \geq 0$ for $t=1, \ldots, n$. 
(2) If $(-1)^{t}\left|A_{t}(x)\right|>0$ for all $t \in\{1, \ldots, n\}$, then $f$ is quasi concave on $T$.

\section{Proof of part 1}

Suppose first that $f$ is a quasi-concave and $C^{2}$ function on the open and convex set $T \subset \square^{n}$. Pick any $x \in T$. If $\nabla f(x)=0$ then we have $\left|A_{t}(x)\right|=0$ for each $t$, since the first row of this matrix is null. This evidently implies $(-1)^{t}\left|A_{t}(x)\right| \geq 0$ for all $t$, therefore part 1 of the theorem is true.

Now, suppose $\nabla f(x) \neq 0$. Define g by $g(y)=\nabla f(x)(x-y)$ for $y \in \square^{n}$, and consider the problem of maximizing $f$ over the constraint set.

$$
C[x]=\left\{y \in \square^{n} \mid g(y) \geq 0\right\}
$$

Since the constraint function is linear, $C[x]$ is a convex set. Since the objective function $f$ is quasi-concave by hypothesis. By the theorem of Kuhn- Tucker that says any point $(y, \lambda)$ meeting the first order conditions identifies a solution to the problem, provided $\nabla f(y) \neq 0$.

We will show that for $\lambda=1$, the pair $(x, \lambda)$ meets the Kuhn-Tucker first order conditions. Since we are assuming that $\nabla f(x) \neq 0$, this means $x$ is a global maximum of $f$ on $C[x]$.Indeed, since $\nabla g(y)=-\nabla f(x)$ at each $y$, we have at $(y, \lambda)=(x, 1)$ $\nabla f(y)+\lambda \nabla g(y)=\nabla f(x)+(-\nabla f(x))=0$

Since $\lambda=1$, it is also satisfies $\lambda \geq 0$. Finally, at $y=x$, we have, $g(y)=0$, so $\lambda(g(y))=0$. Thus, $x$ is a global maximum of $f$ on $C[x]$

Now, define the subset $C^{\prime}[x]$ of $C[x]$ by $C^{\prime}[x]=\left\{y \in \square^{n} \mid g(y)=0\right\}$

Since $x \in C^{\prime}[x]$ and $x$ maximizes $f$ over all $C[x]$, it also maximizes $f$ over $C^{\prime}[x]$. Since $\nabla g(x)=\nabla f(x) \neq 0$, we must have $\rho(\nabla g(x))=1$.

Where $\rho(\nabla g(x))=1$, is the rank of the gradient of $f$ at $x$. Since the constraint qualification is met, $x$ must be the case that the quadratic form $\nabla^{2} f(x)+\lambda \nabla^{2} g(x)$ is negative semi-definite on the $\operatorname{set}\{z \mid \nabla g(x) z=0\}$.

A necessary conditions for negative semi-definiteness to obtain is that the determinants of the sub-matrices $\mathrm{M}_{\mathrm{t}}$ derived from the following matrix by retaining only the first $(t+1)$ rows and columns should have the same sign as $(-1)^{t}, t=1, \ldots, n$ :

$$
M=\left[\begin{array}{cc}
0 & \nabla g(x) \\
\nabla g(x)^{t} & \nabla^{2} f(x)+\lambda \nabla^{2} g(x)
\end{array}\right]
$$

Since $\nabla^{2} g(x)=0$, and $\nabla g(x)=-\nabla f(x)$, the matrix $M_{t}$ is precisely $A_{t}(x)$. Thus, we must have $(-1)^{t}\left|A_{t}^{(x)}\right| \geq 0$ for $k=2, \ldots, n$, and this establishes the first part of the theorem.

The second part of the theorem will be proved using a three-step procedure. Fix an arbitrary point $x \in T$, and let $g(y)=\nabla f(x)(x-y)$.

Step 1, we show that $x$ is itself a strict local maximum of $f$ on the constraint set 
$C^{1}[x]=\{y \in T \mid g(y)=0\}$

Step 2, we show that $x$ is actually a global maximum of $f$ on the constraint set. $C[x]=\{y \in T: g(y) \geq 0\}$. Lastly, we show that $\mathrm{y}$ is any other point in $T$ and $\lambda \in(0,1)$, then $f(\lambda x+1-\lambda) y \geq \min \{f(x), f(y)\}$. Since $x$ was chosen arbitrarily, therefore $f$ is quasiconcave.

So fix $x \in T$, we show that $x$ is a strict local maximum of $f$ on $C^{1}[x]$ by showing (a) that the constraint qualification is met at $x$, (b) that there is $\lambda$ such that $(x, \lambda)$ also meets the first order conditions of the theorem of Lagrange, and (c) that $(x, \lambda)$ also meets the second conditions of Kuhn-Tucker theorem and for a strict local maximum. First, note that $(-1)^{t}\left|A_{t}(x)\right|>0$ at each $t$, we cannot have $\nabla f(x)=0$. Since $\nabla g(y)=-\nabla f(x)$ at any $y \in \square^{n}$ and $\nabla f(x) \neq 0$, we must have $\rho(\nabla g(y))=1$ at all $\mathrm{y}$, and in particular, $\rho(\nabla g(y))=1$. This establishes (a) moreover, for $\lambda=1$, the pair $(x, \lambda)$ is a critical point of the Lagrangian $L(y)=f(y)+\lambda g(y)$, since $\nabla L(x)=\nabla f(x)+\lambda \nabla g(x)=\nabla f(x)-\nabla f(x)$. This establishes (b) lastly, since $\mathrm{g}$ is linear, we have $\nabla^{2} L(x)=\nabla^{2} f(x)$. From the definition of $\mathrm{g}$, it now follows that the condition $(-1)^{t}\left|A_{t}(x)\right|>0, t=1, \ldots, n$, this establishes (c), which proves that $x$ is a strict local maximum of $f$ on $C[x]$, that ends step1.

Proof of step 2: Pick any $y \in[x]$. It suffices to show that $f(x) \geq f(y)$. Since y was choosing arbitrarily in $C[x]$.

Let $x(\alpha)=\alpha x+(1-\alpha) y$ and define the function $F$ on $[0,1]$ by $F(\alpha)=f(x(\alpha))$.

Note that $F(0)=f(y), F(1)=f(x)$, and that $F$ is a $C^{1}$ on $[0,1]$ with $F^{\prime}(\alpha)=\nabla f(x(\alpha))(x-y)$.

Let $\mathrm{C}$ denote the set of minimizers of $F$ on $[0,1]$. and let $\alpha^{*}=\inf C$. We claim that $\alpha^{*}=0$

Suppose $\alpha^{*} \neq 0$. We show the existence of a contradiction. If $\alpha^{*} \in(0,1)$, then we must, of course, have $F^{\prime}\left(\alpha^{*}\right)=0$, since $\alpha^{*}$ is an interior minimum of $\mathrm{F}$ on $[0,1]$; this implies we must have $\nabla f\left(x\left(\alpha^{*}\right)\right)(x-y)=0$. If $\alpha^{*}=1$, then we must have, $F^{\prime}\left(\alpha^{*}\right) \leq 0$, or $\alpha^{*}$ could not be a minimum of $\mathrm{F}$. Therefore, we must have

$\nabla f\left(x\left(\alpha^{*}\right)\right)(x-y) \leq 0$.

However, at $\alpha^{*}=1$ we also have $x\left(\alpha^{*}\right)=x$, and since $\mathrm{y}$ is in $C[x]$, we must have $g(y)=\nabla f(x)(x-y) \geq 0$

Combining (1.15) and (1.16), we have

$\nabla f\left(x\left(\alpha^{*}\right)\right)(x-y)=0$

Now pick any $\xi \in\left(0, \alpha^{*}\right)$, observe that $x\left(\alpha^{*}-\xi\right)-x\left(\alpha^{*}\right)=-\xi(x-y)$, which implies that 


$$
\nabla f\left(x\left(\alpha^{*}\right)\right)\left[x\left(\alpha^{*}-h\right)-x\left(\alpha^{*}\right)\right]=0
$$

By step1 $\nabla f\left(x\left(\alpha^{*}\right)\right)\left[x\left(\alpha^{*}-h\right)-x\left(\alpha^{*}\right)\right]=0$ implies that $x\left(\alpha^{*}\right)$ is a strict local maximum of $f$ on the constraint set

$$
C^{\prime}\left[x\left(\alpha^{*}\right)\right]=\left\{y \in \square^{n} \mid \nabla f\left(x\left(\alpha^{*}\right)\right)\left[x\left(\alpha^{*}\right)-y\right]=0\right\} .
$$

Since $x\left(\alpha^{*}-\xi\right) \in C^{\prime}\left[x\left(\alpha^{*}\right)\right]$ for $\xi \in\left(0, \alpha^{*}\right)$, this means that for $\varepsilon>0$ but sufficiently small, we have $f\left(x\left(\alpha^{*}\right)\right)>f\left(x\left(\alpha^{*}-\xi\right)\right)$. This contradicts the definition of $x\left(\alpha^{*}\right)$ as the smallest minimizer of $\mathrm{F}$ on $C[x]$, and shows that $\alpha^{*} \in(0,1]$ is impossible. Therefore, we must have $\alpha^{*}=0$. Establishing step 2 since $\alpha^{*}=0$ implies that $F(1) \geq F(0)$, which is the same as $f(x) \geq f(y)$.

\section{Proof of step 3:}

Pick any $y \in K$ and $\lambda \in(0,1)$, and let $z=\lambda x+(1-\lambda) y$

Observe that

$$
\nabla f(z) z=\lambda \nabla f(z) x+(1-\lambda) \nabla f(z) y \geq \min \{\nabla f(z) x, \nabla f(z) y\} .
$$

Suppose first that $\nabla f(z) x \leq \nabla(z) y$. Then $\nabla f(z) z \geq \nabla f(z) x$ implies $\nabla f(z)(z-x) \geq 0$.

Therefore, $x \in C[z]$,

Where

$$
C(z)=\left\{w \in \square^{n}: \nabla f(z)(z-w) \geq 0\right\} .
$$

By step 2, z maximizes $f$ over $C(z)$, so we must have $f(z) \geq f(x)$.

Now, suppose $\nabla f(z) y \leq \nabla f(z) x$. Then, we must have, $\nabla f(z)(z-y) \geq 0$, so $y \in C[z]$. Since z maximizes $f$ over $C[z]$, in this case we must have $f(z) \geq f(y)$.

Thus, we must have either $f(z) \geq f(x) \operatorname{orf}(z) \geq f(y)$. This implies that. Therefore $f$ is quasi-concave on $\mathrm{K}$.

Example1.4: Let $f: \square_{++}^{2} \rightarrow \square$ be given by

$$
f(x, y)=x^{2} y^{2},(x, y) \in \square_{++}^{2}
$$

The example below illustrates that weak inequality is not sufficient to identify quasi-concavity.

Let $f: \square^{2} \rightarrow \square$ be given by

$$
f(x-y)=(x-2)^{2}(y-2)^{2},(x, y) \in \square_{+}^{2} \text {. }
$$

Equality holds if and only if $x=2$ or $y=2$

Here, the slack inequality condition are met, but $f$ is not quasi-concave on $\square_{+}^{2}$

Thus, weak inequality is not sufficient to establish quasi-concavity of $f$.

It is quite difficult to get simple necessary and sufficient conditions for quasi-concavity in the case where $f$ is twice continuously differentiable (although, it is easy to get sufficient conditions). In order to get necessary and sufficient conditions, we look at the extreme line segments property. 


\subsection{Characterizations of Quasi-concave Function in Terms of Line Segments.}

Here we are interested with a family of functions that lies between the families of quasi-concave and strictly quasi-concave functions subject to some continuity requirements. Now, we recall that we can define quasi-concave functions by the condition

$$
\begin{gathered}
f(y) \geq f(x) \Rightarrow f(\lambda x+(1-\lambda) y) \geq f(x), \text { for every } 0 \leq \lambda \leq 1, \text { and strict if } \\
f(y) \geq f(x) \Rightarrow f(\lambda x+(1-\lambda) y)>f(x), \text { for } x \neq y, \text { and } 0<\lambda<1
\end{gathered}
$$

Definition 1.1: Let $f$ be defined on the convex set. It is said to be semi-strictly quasi-concave if $f(y)>f(x) \Rightarrow f(\lambda x+(1-\lambda) y)>f(x)$, and $0<\lambda<1$. If $f$ is semi-strictly quasi-concave, then $g=-f$ is semi-strictly quasi-convex function.

Theorem1.7. If $f$ is an upper semi-continuous semi-strictly quasi-concave function defined on the convex set $T \subset \square^{n}$, then it is also quasi-concave.

It is clear that strict quasi-concavity implies semi-strict quasi-concavity and quasi-concavity. Semi-strict quasiconcavity implies quasi-concavity provided the function is upper semi-continuous

We actually want to characterize quasi-concave function using extrema properties of the function on line segments. let $T$

Definition1.2: Let $f(x)$ be a function of $\mathrm{n}$ variables defined for $x \in T$ where $T$ is a convex set.

Then $f$ has the line segment minimum property if and only if

$x \in T, y \in T, x \neq y \Rightarrow \min \{f(\lambda x+(1-\lambda) y)\}, 0 \leq \lambda \leq 1$, exists

That is, the minimum of $f$ along any line segment in its domain of definition exists

Theorem 1.8: (Dinh 1998). Let $f$ be a real function on a convex set $T \subset \square^{n}$. Then the following assertions hold true.

(1) $f$ is quasi-concave if and only if its restriction on any closed segment attains its minimum at a segment end;

(2) Assume $f$ is upper semi-continuous on $T$, then $f$ is semi-strictly quasi-concave if and only if its restriction on any closed segment attains a strict minimum at a segment end whenever it is not constant at that point;

(3) $f$ is strictly quasi-concave if and only if its restriction on any closed segment attains a strict minimum at a segment end.

Proof: The assertion $1 \& 3$ are directly obtained using definitions. We now prove assertion 2 . Assume $f$ is semi-strictly quasi-concave. Let $[x, y]$ be any segment in $T$ with $x \neq y$. Assume further that $f$ is not constant on this line segment, that is $f(c) \neq f(y)$, for some $c \in[x, y]$. If $f(x) \neq f(y)$, say $f(x)>f(y)$. By definition,

$f(\lambda x+(1-\lambda) c)>f(x)$

$f(\lambda y+(1-\lambda) c)>f(y)$

For all $\lambda \in(0,1)$. Hence, $x$ and $y$ are strict maxima of $f$ on $[x, y]$.

Conversely, if $f$ is not semi-strictly quasi-concave function, then there can be found $x, y \in T$ and $\lambda \in(0,1) \quad$ such that $f(x)>f(y) \geq f(\lambda x+(1-\lambda) y)$. It follows in found particular that $f$ is not constant on $[x, y]$. By the first part, we may assume $f$ is quasi-concave. Hence $f$ is constant on $[\lambda x+(1-\lambda) y]$ and $x, y$ cannot be strict maxima of $f$ on $[x, y]$.

\subsection{Optimality condition for quasi-concave functions:}

Formally, it was stated that local maxima of quasi- concave functions need not, in general, also be global maxima, it is only when the function involved is strictly quasi-concave that we will establish global maxima. 
Theorem 2.1: Let $f: T \rightarrow \square$ be strictly quasi- concave where $T \subset \square^{n}$ is convex. Then any local maximum of $f$ on $T$ is also a global maximum of $f$ on $T$. Moreover, the set $\arg \max \{f(x), x \in T\}$ of maximizers of $f$ on $T$ is either empty or a singleton.

We define the following before we prove this proposition:

(1) Global maximum: We say that $x \in T$ is a global minimum of $f$ in $T$ if. $f(x) \geq f(y) \forall y \in \square^{\mathrm{n}}$

(2) $\quad x \in T$, is a local maximum of $f$ in $T$ if there exists $r>0$ such

that $f(x) \geq f(x) \forall x \in T \cap B(x, r)$.

Proof

Suppose $x$ is a local maximum of $f$ on $T$, then there exists $r>0$ such that $f(x) \geq f(y)$ for all $y \in B(x, r) \cap T$. If $x$ were not a global maximum of $f$ on $T$.Then there exists $z \in T$ such that $f(z)>f(x)$. Let $y(\lambda)=\lambda x+(1-\lambda) z, \lambda \in(0,1)$. Note that $y(\lambda) \in T$ because $T$ is convex. By the strict quasi- concavity of $f$, we have

$f(y(\lambda)>\min \{f(x), f(z)\}=f(x)$ for any $\lambda \in(0,1)$. But for $\lambda>1-r / d(x, z)$, we have $d(x, y(\lambda))<r$ so, which contradicts the hypothesis that $x$ is a local maximum proving the first part of the proposition.

Proof of second part, let $x$ and y both be maximizers of $f$ on, pick any $\lambda \in(0,1)$ and

let $z=\lambda x+(1-\lambda) y$. Since $T$ is convex, $z \in T$, since $f$ is strictly quasi concave,

$f(z)=f(\lambda x+(1-\lambda) y)>\min \{f(x), f(y)\}=f(x)=f(y)$ and this contradicts the hypothesis

that $x$ and $y$ are maximizers, proving part two of the theorem.

The most significant part of this theorem is that as with strict concavity, strict quasi concavity implies uniqueness of the solution.

Pseudo-concave function refers the property of concave functions which say that $x \in T$ is maximum if and only if $\nabla f(x)=0$ when $f$ is differentiable.

Theorem 2.2: Let $f$ be differentiable on the open convex set $T \subset \square^{n}$. If $f$ is (strictly)

Pseudo-concave and $\nabla f(x)=0$, then $x$ is a (strict) global maximum of $f$ on $T$.

Moreover, if $f$ is continuously differentiable on $T$, then $f$ is (strictly)

Pseudo-concave and $\nabla f(x)=0$, which shows that $x$ is a (strict) local maximum of $f$ on $T$.

Finally, we consider the sufficient condition for quasi-concave functions that involves inequality constraints problem. The main result for the problem is the theorem of Kuhn and Tucker.

Before we state the theorem of Kuhn and Tucker, we state the following lemma;

Lemma 2.1: Let $f$ and $h_{i}(i=1, \ldots, t)$ be $\mathrm{C}^{1}$ quasi-concave functions mapping $P \subset \square^{n}$ into $\square$ where

$P$ is open and convex

Define

$$
T=\left\{x \in P \mid h_{i}(x) \geq 0, i=1, \ldots, t\right\}
$$

Suppose there exist $x^{*} \in T$ and $\lambda \in \square^{t}$ such that the Kuhn-Tucker first order conditions are met, i.e.

$$
\begin{aligned}
& {[K T-1] \quad \nabla f\left(x^{*}\right)+\sum_{i=1}^{t} \lambda_{i} \nabla h_{i}\left(x^{*}\right)=0} \\
& {[K T-2] \quad \lambda_{i} \geq 0, \lambda_{i} h_{i}\left(x^{*}\right)=0, i=1, \ldots, t .}
\end{aligned}
$$

Then for any $y \in T$, we have 
$\nabla f\left(x^{*}\right)\left(y-x^{*}\right) \leq 0$

. Theorem 2.3 (Theorem of Kuhn-Tucker): Let $f$ and $h_{i}(i=1, \ldots, t)$ be $C^{1}$ quasi-concave functions mapping $P \subset \square^{n}$ into $\square$ where $P$ is open and convex.

Define

$$
T=\left\{x \in P \mid h_{i}(x) \geq 0, i=1, \ldots, t\right\} .
$$

Suppose there exists $x^{*} \in T$ and $\lambda \in \square^{t}$ such that the Kuhn-Tucker first order conditions are met:

$[K T-1] \nabla f\left(x^{*}\right)+\sum_{i=1}^{t} \lambda_{i} \nabla h_{i}\left(x^{*}\right)=0$

$[K T-2] \quad \lambda_{i} \geq 0, \lambda_{i} h_{i}\left(x^{*}\right)=0, i=1, \ldots, t$

Then, $x^{*}$ maximizes $f$ over $T$ provided at least one of the following conditions holds

$[Q c-1] \nabla f\left(x^{*}\right) \neq 0$

$[Q c-2] f$, is concave.

For the purpose of this paper we just give a hint for the proof.

Hint: By the hypothesis $\nabla f\left(x^{*}\right)+\sum_{i=1}^{t} \lambda_{i} \nabla h_{i}\left(x^{*}\right)=0$

$\Rightarrow \nabla f\left(x^{*}\right)\left(y-x^{*}\right)=-\sum_{i=1}^{t} \lambda_{i} \nabla h_{i}\left(x^{*}\right)\left(y-x^{*}\right)$

Show that the sum on the right- hand side is non- positive. To show this, it suffices to show that for each $i \in(1, \ldots, t), \lambda_{i} \nabla h_{i}\left(x^{*}\right)\left(y-x^{*}\right) \geq 0$

Then show that the theorem is true under each of the two conditions

$[Q c-1] \nabla f\left(x^{*}\right) \neq 0$

$[Q c-2] f$ is concave.

For the full proof see [3]

\section{Selected references}

[1]. D.T.Luc, Generalized convexity and some Applications to vectors optimization, vietnam journal of Mathematics 26:2(1998)

[2]. D. T. Luc, characterizations of quasi-convex functions, Bulletin of Australia mathematical society 48 (1993).

[3]. Rangarajan, K. Sundaram, A first course in optimization theory, Cambridge, United States; University Press, (1976)

[4]. Schaible S. and Ziemba W.T,Generalized concavity I optimization and Economics,Academic Press,New York, (1981)

[5]. Stephen Boyd and Lieven Vandenberghe, Convex Optimization, Cambridge, Syndicate of the University Press, (2006).

[6]. W.E. Diewert, A.Avriel, and I. Zang, Generalized concavity (2010).

[7]. I.Ginchev and V.I. Ivanov, Second order characterizations of convex and pseudo-convex functions,Journal of Analysis vol.9,No 2 (2003). 